\title{
Principes généraux pour une méthode d'appréciation de la qualité globale des sédiments lacustres à l'aide d'une analyse simplifiée des malacocénoses
}

\author{
J. Mouthon 1
}

Mots clés : Mollusques, lacs, capacité biogénique, sédiments.

Trois phénomènes généraux établis à partir d'observations réalisées sur trente lacs permettent de décrire la dégradation progressive des peuplements de Mollusques. Un modèle composé de sept situations successives et susceptible de fournir les bases d'une méthode globale d'appréciation de la capacité biogénique des sédiments lacustres est proposée.

General principles for a method of assessing the overall quality of lacustrine sediments, using a simplified analysis of the molluse community.

Keywords : Mollusc, lakes, biogenic capacity, sediments.

Three general phenomena established from observations on thirty lakes can be used to describe a progressive degradation in the mollusc populations. A model with seven successive situations is proposed and serves as an overall method of assessment for the biogenic capacity of lacustrine sediments.

\section{Introduction}

Comme pour de nombreuses espèces d'Insectes et notamment les Diptères, certains Crustacés, les Vers..., le développement des Mollusques vivant en milieu lacustre est étroitement dépendant de la qualité des sédiments. C'est le cas pour les Bivalves, à l'exception cependant de Dreissena polymorpha Pal. qui choisit généralement pour se fixer un support solide, mais également pour les Gastéropodes colonisant à nouveau ce substratum, dès que la végétation qu'ils envahissent pendant la saison estivale, commence en début d'automne à se décomposer.

L'étude des peuplements de Mollusques, effectuée au cours de ces dix dernières années sur une trentaine de lacs appartenant à différentes régions fran. çaises, Jura, Alpes, Massif-Central, Vosges, Vendée (Tableau I), a montré que leur distribution dans les zones littorales, sublittorales et profondes

1. CEMAGREF 3, quai Chauveau 69009 LYON présentaient parfois des variations importantes d'un milieu à l'autre, même géographiquement très proches. L'utilisation de ces observations ainsi que la recherche de références malacologiques, de la fin du siècle à nos jours, nous ont conduit à définir trois phénomènes autour desquels s'organise l'évolution des malacocénoses d'un système lacustre.

\section{Matériel et méthode}

L'échantillonnage est généralement réalisé au cours de la saison chaude (de mai à septembre), à raison le plus souvent de deux campagnes par lac.

Les Mollusques de la zone littorale sont récoltés à l'aide d'un troubleau à base rectangulaire $(25 \times$ $18 \mathrm{~cm}$ ) ; la surface prospectée variant de 0,5 à $3 \mathrm{~m}^{2}$ par station suivant la nature des dépôts.

Les zones profondes sont prospectées à l'aide d'une benne Friedinger (surface environ $350 \mathrm{~cm}^{2}$ ), trois à six échantillons étant effectués à chaque point d'échantillonnage, répartis sur deux, trois ou 
Tableau I. Liste des milieux lacustres prospectés.

\begin{tabular}{|c|c|c|c|c|}
\hline & Lacs & Aititude & $\begin{array}{l}\text { Profondeur } \\
\text { maximale (m) }\end{array}$ & $\begin{array}{l}\text { Superficie } \\
\text { (ha) }\end{array}$ \\
\hline JURA & $\begin{array}{l}\text { St Point } \\
\text { Remoray } \\
\text { Abbaye } \\
\text { Les Rousses } \\
\text { Grand Clairvaux } \\
\text { Petit Clairvaux } \\
\text { Grand Maclu } \\
\text { Petit Maclu } \\
\text { Ilay } \\
\text { Chalain } \\
\text { Bonlieu } \\
\text { Grand Etival } \\
\text { Petit Etival } \\
\text { Antre } \\
\text { Narlay } \\
\text { Nantua } \\
\text { Val }\end{array}$ & $\begin{array}{r}849 \\
849 \\
879 \\
1059 \\
534 \\
535 \\
779 \\
779 \\
778 \\
500 \\
803 \\
790 \\
790 \\
824 \\
750 \\
478 \\
522\end{array}$ & $\begin{array}{l}40,3 \\
27,6 \\
19,5 \\
18,2 \\
20 \\
16 \\
25,8 \\
11,5 \\
30 \\
34 \\
16 \\
9,5 \\
7,5 \\
4 \\
40 \\
43 \\
24,5\end{array}$ & $\begin{array}{c}398,2 \\
95,3 \\
92,45 \\
89,8 \\
63,46 \\
17,39 \\
24,4 \\
6,4 \\
72,6 \\
231,8 \\
17,36 \\
16 \\
7,25 \\
41,6 \\
141 \\
49\end{array}$ \\
\hline VOSGES & $\begin{array}{l}\text { Gërardmer } \\
\text { Longemer } \\
\text { Lispach } \\
\text { Lac des Corbeaux } \\
\text { Lac Vert }\end{array}$ & $\begin{array}{r}660 \\
750 \\
905 \\
\simeq 900 \\
1044\end{array}$ & $\begin{array}{r}36 \\
30 \\
\simeq 5 \\
23 \\
\simeq 20\end{array}$ & $\begin{array}{r}115,5 \\
76,2 \\
9,2\end{array}$ \\
\hline ALPES & $\begin{array}{l}\text { Grand Ban } \\
\text { Lac Rond } \\
\text { Lac du Serpent } \\
\text { Lac Laramon } \\
\text { Tignes } \\
\text { Leman (Secteur: Nernier, } \\
\text { Thonon, Sciez, Yvoire) }\end{array}$ & $\begin{array}{r}2450 \\
2380 \\
2450 \\
2360 \\
2086 \\
\quad 372\end{array}$ & $\begin{array}{r}10 \\
9 \\
10 \\
12 \\
\simeq 43 \\
309\end{array}$ & $\begin{array}{c}32,28 \\
582,4\left(\mathrm{~km}^{2}\right)\end{array}$ \\
\hline Massif-Central & Aydat & 825 & 1515 & 60,31 \\
\hline Vendēe & $\begin{array}{l}\text { Lac de Grandlieu } \\
\text { (bordure orientale) }\end{array}$ & 2 & $\simeq 5$ & 3600 \\
\hline
\end{tabular}


quatre transects, choisis en fonction des caractéristiques morphologiques de chaque plan d'eau.

\section{Définition des 3 phénomènes carac- térisant les différents stades d'évolu- tion des Malacocénoses}

\subsection{Premler phénomène: Colonisation profonde potentielle.}

"L'existence d'une faune profonde paraissant jusqu'alors une impossibilité "(Favre 1940), ce n'est qu'à la fin du siècle dernier que l'on a commencé à prospecter les fonds des grands lacs. Les recherches des pionniers de la limnologie que furent Clessin, Forel, Naumann, Imhof, Pauli, Thienemann, Wesenberg-Lund... révélèrent alors l'existence d'une macrofaune profonde relativement riche dominée par les Diptères, les Vers et les Mollusques.

Malgré leur imprécision et leur caractère fragmentaire, les résultats de ces premières recherches constituent actuellement la seule référence fiable, les données paléontologiques demeurant peu accessibles au niveau des zones profondes, permettant de connaitre l'amplitude bathymétrique optimale des espèces de Mollusques en milieu lacustre.

La lecture du tableau 2 où seuls les trois genres les plus eurybathes ; Lymnaea, Valvata et Pisidium sont pris en considération montre que :

- Gastéropodes et Bivalves colonisaient autrefois la zone profonde de plusieurs grands systèmes lacustres ; ce sont, parmi les milieux ayant fait l'objet de recherches, les lacs Léman, Quatre Cantons, Walen, Annecy, et probablement Windermere.

- Les Pisidies constituaient à une époque encore récente un élément permanent de la faune de fond des lacs. En ce qui concerne le Léman (Mouthon, sous presse) et le Lac d'Annecyl, ce n'est aujourd'hui plus le cas.

Comme le faisait déjà remarquer Alsterberg en 1930, les dragages réalisés dans de nombreux autres systèmes lacustres comme les lacs de Zurich, Bour. get, Majeur, Thoune... (cf. p. 76 et 77) n'ont pas permis la récolte de Gastéropodes. Toutefois on remarquera que si dans plusieurs lacs les genres Lymnaea et Valvata ne dépassent guère les zones littorales et

\footnotetext{
1. Données Juget \& Lafont 1973-1974, non publiées.
}

sublittorales, dans d'autres milieux ces espèces atteignent des profondeurs plus importantes, situées bien au-delà de la thermocline (Tableau II). Une explication à ces différents modèles de distribution réside sans doute, dans l'apparition à titre transitoire ou permanent de conditions inadéquates au niveau des fonds contraignant les Gastéropodes à déserter cette zone, et réduisant ainsi leur amplitude bathymétrique.

Gastéropodes et Bivalves sont donc théoriquement capables de coloniser la zone profonde des écosystèmes lacustres, lorsque les conditions sont favorables.

\subsection{Deuxième phénomène : désertion progressive des sédiments profonds.}

Déjà en 1917, Wesenberg-Lund attribuait la faible amplitude bathymétrique et la pauvreté du benthos de la zone profonde des lacs baltiques, aux faibles concentrations en oxygène dissous régnant au niveau des fonds pendant la saison estivale.

Dans un nombre d'écosystèmes sans cesse en augmentation, les modifications de l'amplitude bathymétrique des espèces constatées par de nombreux auteurs, sont généralement attribuées à un accroissement d'apports polluants et inhibiteurs (Bagge \& Jumppanen 1968, Ruggiu \& Saraceni 1972, Jumppanen 1976, Bianchi \& Ravera 1977, Girod \& Kuiper 1977, Kansanen \& Aho 1981). Ce phénomène a également été constaté dans le Lac Léman où des prélèvements récents (Mouthon, sous presse) ont révélé la disparition des limnées et des pisidies de la plaine centrale, réduisant notablement l'amplitude bathymétrique de ces espèces.

Lorsque les conditions du milieu se dégradent, on assiste à une réduction de l'amplitude bathymétrique des organismes vivant ordinairement au niveau des fonds en direction des zones sublittorales, puis littorales, et corrélativement à la disparition de certaines especes, comme Pisidium conventus et $P$. personatum inféodées aux zones profondes des milieux lacustres d'Europe moyenne.

\subsection{Troisième phénomène : modifications inverses des populations de Bivalves et de Gastéropodes en zones littorale et profonde.}

De nombreux prélèvements effectués dans la zone littorale des différents lacs étudiés, dont certains 
Tableau II. - Profondeur atteinte par les genres Lymnaea, Valvata et pisidium dans différents lacs européens.

(1) Alsterberg (1930) ; (2) Forel (1885) ; (3) Zschokke (1911); (4) Absence de données au-delà de cette profondeur

\begin{tabular}{|c|c|c|c|c|c|}
\hline \multirow{2}{*}{$\begin{array}{l}\text { Lac } \\
\text { LOMOND }\end{array}$} & \multirow{2}{*}{$\begin{array}{r}\text { (Pays) } \\
\text { (GB) }\end{array}$} & \multirow{2}{*}{$\begin{array}{c}\begin{array}{c}\text { Profondeur } \\
\text { maximale }(\mathrm{m})\end{array} \\
198\end{array}$} & \multicolumn{2}{|c|}{$\begin{array}{l}\text { Profondeur atteinte par } \\
\text { les mollusques cités (m) }\end{array}$} & \multirow{2}{*}{$\begin{array}{r}\text { Référence } \\
\text { Slack }(1966)\end{array}$} \\
\hline & & & Pisidium & 198 & \\
\hline NESS & (GB) & 230 & Pisidium & 230 & Murray et Pular (1910) \\
\hline WINDERMERE & (GB) & 67 & $\begin{array}{l}\text { Lymnaea } \\
\text { Valvata } \\
\text { Pisidium }\end{array}$ & $\begin{array}{l}56 \\
9 \\
60(4)\end{array}$ & $\begin{array}{l}\text { Humphies (1936) } \\
\text { Macan (1950) }\end{array}$ \\
\hline BRACCIANO & (I) & 165 & Pisidium & 165 & Bonomi \& Ruggiu (1971) \\
\hline BOLSENA & (I) & 151 & Pisidium & 151 & \\
\hline COME & (I) & 409 & $\begin{array}{l}\text { Lymnaea } \\
\text { Pisidium }\end{array}$ & $\begin{array}{l}100(4) \\
100(4)\end{array}$ & $\begin{array}{l}(2) \\
(2)\end{array}$ \\
\hline GARDE & (I) & 346 & Valvala & $90(4)$ & Clessin $(1890)$ \\
\hline LUGANO & (I) & 288 & $\begin{array}{l}\text { Lymnaea } \\
\text { Pisidium }\end{array}$ & $\begin{array}{r}90 \\
288\end{array}$ & $\begin{array}{c}(1) \\
\text { Fehlman (1911) }\end{array}$ \\
\hline MAJEUR & (I) & 372 & Pisidium & $300(4)$ & $(2)$ \\
\hline MERGOZZO & (I) & 74 & $\begin{array}{l}\text { Lymnaea } \\
\text { Valvata } \\
\text { Pisidium }\end{array}$ & $\begin{array}{l}10 \\
20 \\
70(4)\end{array}$ & Bonomi et Ruggiu (1966) \\
\hline VICO & $(\mathbf{I})$ & 48,5 & Pisidium & 48,5 & Bonomi \& Ruggiu (1971) \\
\hline GOKTSCHAI & (TU) & 110 & Lymnaea & $72(4)$ & $(3) \cdot(1)$ \\
\hline KILPISJARVI & (FL) & 50 & $\begin{array}{l}\text { Lymnaea } \\
\text { Pisidium }\end{array}$ & $\begin{array}{l}21 \\
50\end{array}$ & Bagge (1969) \\
\hline KONNEVESI & $(\mathbf{F L})$ & 49 & Pisidium & $45(4)$ & Särkkä (1972) \\
\hline ANKAVATTNETT & (S) & 70 & $\begin{array}{l}\text { Lymnaea } \\
\text { Pisidium }\end{array}$ & $\begin{array}{r}5 \\
70\end{array}$ & Grimas (1961) \\
\hline BALSJÖN & (S) & 147 & $\begin{array}{l}\text { Lymnaea } \\
\text { Pisidium }\end{array}$ & $\begin{array}{r}13 \\
147\end{array}$ & Grimas (1961) \\
\hline $\begin{array}{l}\text { TORNE-TRÄSK } \\
\text { VATTERN }\end{array}$ & $\begin{array}{l}\text { (S) } \\
\text { (S) }\end{array}$ & $\begin{array}{l}130 \\
128\end{array}$ & $\begin{array}{l}\text { Pisidium } \\
\text { Lymnaea } \\
\text { Valvata } \\
\text { Pisidium }\end{array}$ & $\begin{array}{r}130 \\
55 \\
34 \\
120\end{array}$ & $\begin{array}{l}\text { Steenberg }(1917) \\
\text { Ekman }(1915.1916)\end{array}$ \\
\hline ESROM & (DK) & 22 & $\begin{array}{l}\text { Lymnaea } \\
\text { Valvata } \\
\text { Pisidium }\end{array}$ & $\begin{array}{l}11 \\
20 \\
22\end{array}$ & Berg (1938) \\
\hline FURE & (DK) & 36 & $\begin{array}{l}\text { Lymnaea } \\
\text { Valvata } \\
\text { Pisidium }\end{array}$ & $\begin{array}{r}8 \\
13 \\
36\end{array}$ & Steenberg (1917) \\
\hline CONSTANCE & (D) & 252 & $\begin{array}{l}\text { Lymnaea } \\
\text { Valvata } \\
\text { Pisidium }\end{array}$ & $\begin{array}{r}70 \\
50 \\
252\end{array}$ & (1) \\
\hline PLONN & (D) & 75 & Pisidium & 75 & (B) \\
\hline STARNBERG & (D) & 245 & $\begin{array}{l}\text { Lymnaea } \\
\text { Pisidium }\end{array}$ & $\begin{array}{l}80(4) \\
60(4)\end{array}$ & a \\
\hline ACHEN & (A) & 134 & $\begin{array}{l}\text { Lymnaea } \\
\text { Valvata }\end{array}$ & $\begin{array}{l}72(4) \\
64(4)\end{array}$ & $\begin{array}{l}\text { (3) }-(1) \\
\text { (3) }\end{array}$ \\
\hline LUNZER & (A) & 33 & Pisidium & $30(4)$ & Hadl (1972) \\
\hline LEMAN (CH-F) & & 309 & $\begin{array}{l}\text { Lymnaea } \\
\text { Valvata } \\
\text { Pisidium }\end{array}$ & $\begin{array}{l}309 \\
100 \\
309\end{array}$ & $\begin{array}{l}\text { (2) } \\
\text { (3) } \\
\text { (1) }\end{array}$ \\
\hline NEUCHATEL & (CH) & 153 & $\begin{array}{l}\text { Lymnaea } \\
\text { Pisidium }\end{array}$ & $\begin{array}{r}73 \\
144\end{array}$ & $\begin{array}{c}\text { (1) } \\
\text { Favre (1941) }\end{array}$ \\
\hline
\end{tabular}


Tableau II. (suite)

\begin{tabular}{|c|c|c|c|c|c|}
\hline \multirow{2}{*}{$\begin{array}{l}\text { Lac } \\
\text { THOUNE }\end{array}$} & \multirow{2}{*}{$\begin{array}{r}\text { (Pays) } \\
(\mathrm{CH})\end{array}$} & \multirow{2}{*}{$\begin{array}{c}\begin{array}{c}\text { Profondeur } \\
\text { maximale (m) }\end{array} \\
217\end{array}$} & \multicolumn{2}{|c|}{$\begin{array}{l}\text { Profondeur atteinte par } \\
\text { les mollusques cités (m) }\end{array}$} & \multirow{2}{*}{$\begin{array}{c}\text { Référence } \\
\text { (3) } \\
\text { (3) }\end{array}$} \\
\hline & & & $\begin{array}{l}\text { Valvata } \\
\text { Pisidium }\end{array}$ & $\begin{array}{c}45 \\
100(4)\end{array}$ & \\
\hline VIERWALDSTATT & $(\mathrm{CH})$ & 214 & $\begin{array}{l}\text { Lymnaea } \\
\text { Pisidium }\end{array}$ & $\begin{array}{l}200 \\
214\end{array}$ & $\begin{array}{c}\text { (1) - (2) } \\
\text { (3)-Steenberg (1917) }\end{array}$ \\
\hline WALEN & $(\mathrm{CH})$ & 151 & $\begin{array}{l}\text { Lymnaea } \\
\text { Pisidium }\end{array}$ & $\begin{array}{l}150 \\
150\end{array}$ & $\begin{array}{l}(3)-(1) \\
(3)\end{array}$ \\
\hline WETTER & $(\mathrm{CH})$ & 120 & Lymnaea & $56(4)$ & (1) \\
\hline ZOUG & $(\mathrm{CH})$ & 198 & Pisidium & 198 & (3) \\
\hline ZURICH & (CH) & 140 & $\begin{array}{l}\text { Lymnaea } \\
\text { Valvaia } \\
\text { Pisidium }\end{array}$ & $\begin{array}{r}40 \\
40 \\
140\end{array}$ & $\begin{array}{c}\text { (3) } \\
\text { (3) } \\
\text { (3)-Lundbeck (1926) }\end{array}$ \\
\hline ANNECY & (F) & 65 & $\begin{array}{l}\text { Lymnaea } \\
\text { Valvata } \\
\text { Pisidium }\end{array}$ & $\begin{array}{l}65 \\
40 \\
65\end{array}$ & $\begin{array}{l}(2) \\
(3) \\
(2)\end{array}$ \\
\hline BOURGET & (F) & 145 & $\begin{array}{l}\text { Lymnaea } \\
\text { Valvata } \\
\text { Pisidium }\end{array}$ & $\begin{array}{r}45 \\
25 \\
145\end{array}$ & $\begin{array}{c}\text { Favre (1940) } \\
\text { (2) }\end{array}$ \\
\hline LONGEMER & (F) & 29,5 & Pisidium & 29,5 & $\begin{array}{c}\text { Meier-Brook (1963) } \\
\text { Rapport CTGREF (1979) }\end{array}$ \\
\hline CHALAIN & (F) & 34 & $\begin{array}{l}\text { Lymnaea } \\
\text { Valvala } \\
\text { Pisidium }\end{array}$ & $\begin{array}{r}3 \\
10 \\
34\end{array}$ & $\begin{array}{c}\text { Rapport SRAE } \\
\text { Franche-Comté(1986) }\end{array}$ \\
\hline
\end{tabular}

(lacs de Clairvaux, lac de l'Abbaye) ont été réalisés à l'aval de rejets inhibiteurs (Rapport SRAE 1984), ou à proximité des embouchures d'afférences polluées (Mouthon 1981b), nous ont permis de préciser les modalités de la simplification progressive de ces malacocénoses ; 9 stades illustrés d'exemples ont pu ainsi être distingués (fig. 1).

Caractérisé par une richesse faunistique élevée, et une bonne répartition des abondances aussi bien chez les Gastéropodes que chez les Bivalves, ce sont les malacocénoses peuplant la bordure septentrionale du lac St Point qui ont été retenues comme référence (situation 1 ).

Les premiers stades (2 à 4) montrent :

- une baisse sensible du nombre des espèces, mais une augmentation de l'abondance globale (stades 2 et 3), puis une nette diminution de cette dernière (stade 4).

- la prédominance des Planorbidae dont les représentants sont généralement peu exigeant vis à vis des concentrations en oxygène dissous du milieu (Germain 1931, Boycott 1936).
- parmi les Bivalves, la nette diminution de Pisidium subtruncatum sans doute la plus commune, et la plus polluorésitante des pisidies (Mouthon, 1981a).

Puis interviennent :

- une baisse notable de la richesse spécifique jusqu'au stade 7 et de l'abondance globale (stade 9).

- la disparition totale des Bivalves appartenant au genre Pisidium.

Une hypothèse de travail pouvant être retenue se fonde sur la constatation que l'on observe ce phénomène le plus souvent au voisinage de rejets locaux, et dans certains cas pour l'ensemble des malacocénoses d'un plan d'eau, lorsque la pollution devient chronique comme dans les lacs de Nantua (Vivier \& Serruya 1966, Feuillade 1985), et de Narlay (Masson \& al. 1978).

On peut retenir que l'augmentation des teneurs en matières organiques favorise les espèces les plus saprophiles en fonction des habitats présents :

- Planorbidae et Pisidium subtruncatum pour les bordures riches en débris organiques et (ou) à 
$1 \quad$ Lac de St Point ( 582 ind $/ \mathrm{m}^{2}$ )

2 ( 795 ind $/ \mathrm{m}^{2}$ )

3 Lac de Bonlieu $\left(715\right.$ ind $\left./ \mathrm{m}^{2}\right)$

$4 \quad$ Lac d'Aydat

5 Lac de Clairvaux ( 158 ind $/ \mathrm{m}^{2}$ )

6 Lax de l'Abbaye ( 162 ind $\left./ \mathrm{m}^{2}\right)$

7 Lax de Narlay

( $59 \mathrm{ind} / \mathrm{m}^{2}$ )

- Lar de Nantua ( $41 \mathrm{ind} / \mathrm{n}^{2}$ )

g Lae de Clairvanx $\left(28 \mathrm{nd} / \mathrm{m}^{2}\right)$
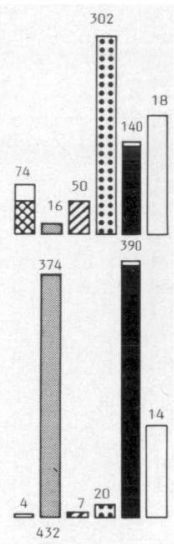

SENS DE SIMPLIFICATION CROISSANTE DES MALACOCENOSES LITTORALES

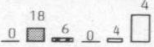

Pt PI Pu Pi Ab Rs
Bilhyna tent aculata Prosobranches(opetcutess) - Fr

Plenorbidee - Pl

Auties pulmones - Pu

Pisidies saut $P$. subtruncotum - Pi

Pisidium subtruncetum -

Aurtes bivalves - $A D$ -

Richesse spécifique - Rs -

Fig: 1. Dégradation croissante des malacocénoses littorales. 
sédiments fins, souvent associés à une végétation clairsemée composée de Phragmites, Scirpes et Nénuphares (Mouthon 1981b).

- Bithynia tentaculata et Sphaerium corneum lorsque le substratum est plus grossier (cailloux, dépôts sableux) comme dans le lac de Nantua où ces deux espèces proliféraient avant que les rejets ne deviennent trop inhibiteurs (Léger 1928). Actuellement, seuls quelques spécimens de Bithynia tentaculata colonisent encore la zone littorale de ce lac, et Sphaerium comeum a presque totalement disparu*. Ce sont d'ailleurs ces espèces qui constituent l'association dominante des secteurs de cours d'eau pollués appartenant au rhithral (Mouthon 1980, 1981a).

Dans les zones littorales des milieux pollués étudiés, mais ce fut peut-être le cas dans le lac de Nantua (Léger 1928), je n'ai pas constaté de réel remplacement des planorbidae par Bithynia tentaculata comme Harman et Forney (1970) l'on décrit pour des espèces américaines du lac Oneida affecté par une pollution chronique.

Dans les zones littorales présentant une dégradation accentuée de leur peuplement, les Gastéropodes apparaissent plus polluorésistants que les pisidies particulièrement lorsque l'effet inhibiteur s'accentue. En revanche dans les zones profondes de certains lacs où vivent des Gastéropodes (Lymnaea), ceux-ci apparaissent nettement plus sensibles que les pisidies aux conditions d'oxygénation régnant à ce niveau (tableau 2), comme l'ont mont ré les résultats de prélèvements quantitatifs effectués par Juget dans le Lac Léman au cours des années 1959 à 1963, et leur confrontation aux données actuelles (Mouthon, sous presse). Ainsi, pendant les années 1961 et 1962, et à la suite de l'apparition d'un déficit important de la concentration en oxygène des eaux profondes (Monod \& al. 1984), on enregistra une dis. parition quasi totale des Lymnaea dans la zone profonde (Isobathes 30 à $100 \mathrm{~m}$ ), tandis que les Sphaeriidae atteignaient aux mêmes profondeurs des densités bien supérieures à celles des années 1959 et 1960. A l'heure actuelle Lymnaea et pisidies ont déserté totalement la plaine centrale de ce lac et l'amplitude bathymétrique de ces Bivalves $(0$ à

\footnotetext{
- Campagne réalisée en aoút 1984, donnès non publiées.
}

$200 \mathrm{~m}$, et sans doute davantage $)^{\star \star}$ est demeurée supérieure à celle des Gastéropodes $(0$ à $170 \mathrm{~m})$.

Lorsque les conditions du milieu se dégradent, les Gastéropodes disparaissent de la zone profonde bien avant les pisidies. Au contraire, dans la zone littorale ce sont préférentiellement les Bivalves qui disparaissent avant les Gastéropodes.

\section{Proposition d'un modèle et discussion}

Les différents modes de distribution des malacocénoses, observés dans les milieux lacustres étudiés, nous ont conduit à la définition de 3 phénomènes permettant de décrire la dégradation progressive des peuplements de Mollusques.

A partir de ces 3 observations est proposé un modèle de modification progressive théorique des peuplements composé de sept situations successives, illustrées d'exemples choisis parmi les milieux étudiés (fig. 2). Ces différents états représentent l'évolution des malacocénoses d'un milieu lacustre très favorable à un écosystème de moins en moins apte au développement des Mollusques, le passage d'un stade théorique à un autre correspondant à une diminution de l'amplitude bathymétrique des espèces, et à une simplication croisssante des malacocénoses.

L'aptitude d'un écosystème à héberger une faune abondante et richement diversifiée est généralement admise comme une expression globale de sa qualité ; ce type de modèle est susceptible de fournir les bases d'une méthode globale d'appréciation de la capacité biogénique des sédiments lacustres, contribuant par là à une meilleure connaissance de la qualité biologique du milieu.

Afin de permettre l'élaboration d'une telle méthode, nous nous proposons au cours de travaux ultérieurs, de définir rationnellement à l'aide d'outils mathématiques appropriés l'ensemble des faits biocénotiques observés, puis de rechercher et d'analyser les causes naturelles et provoquées de telles situations. Parmi celles-ci, la connaissance des conditions physico-chimiques régnant au sein des sédiments, notamment au voisinage interface eausédiment (Verneaux \& al., sous presse), ainsi que

\footnotetext{
* " Les teneurs en oxygène relevèes ces dernières années mont rent que c'est seulement à partir de l'Isobathe $250 \mathrm{~m}$ que les concentrations deviennent limitantes.
} 


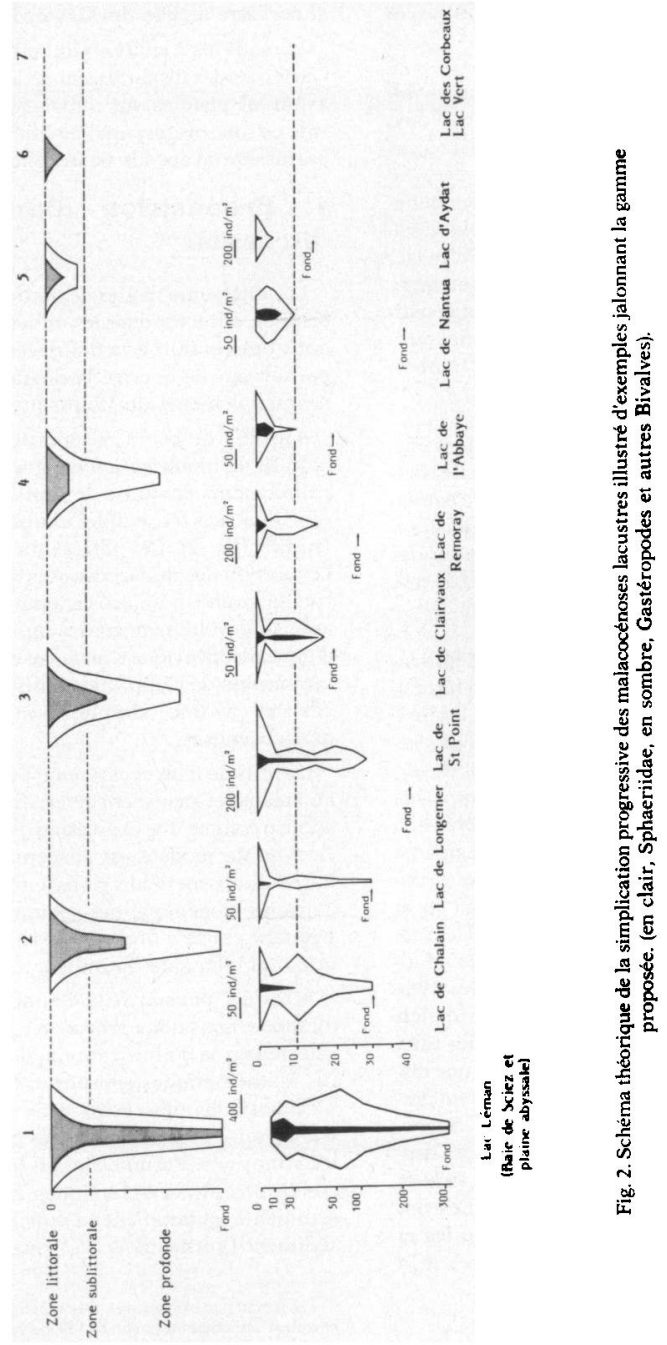


dans la masse d'eau elle-même, la présence ou non d'éléments inhibiteurs apparaissent essentielles.

\section{Remerclements}

L'auteur remercie Messieurs J. Verneaux (Université de Besançon) et $\mathbf{J}$. Juget (Université Lyon I) qui ont bien voulu critiquer ce manuscrit, ainsi que Madame Taillole qui en a assuré la dactylographie.

\section{Travaux cites}

Alsterberg (G.). 1930. - Wichtige Züge in der Biologie der Süsswassergastropoden. Lund (A.B. Gleerlupska Unit. Bokhandeln) $130 \mathrm{pp}$.

Bagge (P.) \& Jumppanen (K.). 1968. - Bottom fauna of lake Kei tele, Central Finland, in relation to hydrography and eutrophi zation. Ann. Zool. Fennici, $5: 327-337$.

Bagge (P.). 1969. - Ecological studies on the fauna of subarctic waters in Finnish Lapland. Ann. Univ. Turku A.II : 40 (Rep. Kevo Subarct. res. Station), $4: 32.83$

Berg (K.). 1938. - Studies on the bot tom animals of Esrom Lake $K$. danske Vidensk. Selk. Natun. Malh. Afd, 8: 1-255.

Bianchi (1.) \& Ravera (O.). 1977, - Ricerche sui Molluschi del lago del Piano (Como). Atti. Soc. ital. Sci. nat. Museo Civ. Stor. nat. Milano, 118, (2): 285-290

Bonomi (G.) \& Ruggiu (D.). 1966. - Il macrobenton profondo del lago di Mergozzo. Memorie Ist. ital. Idrobiol., $20: 153-200$

Bonomi (G.) \& Ruggiu (D.). 1971. - Popolamenti bentonici profondi. In : Limnologia ed ecologia dei Laghi di Bolsena, Brac. ciano, Trasimento e vico : situazione artuale e prevedibili conseguenze derivanti da una loro ulilizzazione multipla. Rapporto finale, Istituto italiono di Idrobiologia, Pallanza : 227-248.

Clessin (S.). 1890. - Molluskenfauna Osterreich-Ungarms und Schweiz, Nümberg.

Ekman (S.). 1915-1916. - Die Bodenfauna des Vättern, qualita. tiv und quantitativ Untersucht. Int. Revue ges. Hydrobiol. Hydrogr., $7: 146-425$.

Favre (J.). 1940. - La faune malacologique post-glaciaire et actuelle du lac du Bourget. Ann. Ecole Nat. Eaux et Forêts, $?$ (2) : 294444

Favre (J.). 1941. - Les Pisidium du canton de Neuchatel. Soc. Neuch. Sci. Nat. $66: 57-112$

Fehlmann (W.). 1911. - Die Tiefenfauna des Luganer Sees. Int. Rev. ges. Hydrob. Hydrol., Suppl., 5 : $1-52$

Feuillade (J.). 1985. - Caracterisation et essais de restauration d'un ecosystème dégradé : le lac de Nantua. J. Feuillade ed., Institut National de la Recherche Agronomique Thonon-les-Bains. Paris : 165 p.

Forel (F.A.). 1885. - La faune profonde des lacs suisses. Notiveaux mémoires de la Soc. helv. de Sci. Nat.

Girod (A.) \& Kuiper (J.G.J.). 1977. - Notes sur les Sphaeriidae du lac de Lugano. Atti Soc, ital. sci. nat. Museo Civ. Stor, nat. Milano. $118: 293-298$

Grimas (U.). 1961. - The bottom fauna of natural and impounded lakes in northern-Sweden (Ankarvattnet and Bläsjön). Imst. Freshw. Research. Report., 42 : 183-237.

Hadl (G.). 1972. - Zür Okologie und Biologie der Pisidien (Bival via : Sphaeriidae) in Lunzer Untersee. Sber äst. Akad. Wiss. math.nat. kl., Abt. I (180) : 317.338.

Harmann (W.N.) \& Formey (J.L.) 1970. - Fifty years of change in the Molluscan fauna of Oneida Lake, New-York. Limnol. Ocea. nogr., $15: 454-460$.

Humphries (C.F.). 1936. - An investigation of the profundal and sublittoral fauna of Windermere. J. Anim. Ecol, $5: 29-52$.
Jumppanen (K.). 1976. - Effects of waste waters on lake ecosystem. Ann. Zool. Fennici, 13: 85-138.

Kansanen (P.K.) \& Aho (J.). 1981. - Changes in the macrozoobenthos associations of polluted lake Vanajavesi, Southern Finland, over a period of 50 years. Ann. Zool. Fennici $18: 73.101$.

Leger (L.). 1927. - Etude hydrobiologique pour servir à l'aména. gement et à la restauration piscicole du Lac de Nantua. Trav. Lab. d'Hydrob. Pisc. Grenoble., $12: 5.78$.

Lundbeck (J.). 1926. - Die Bodentierwelt Norddeutscher Seen. Arch. Hydrobiol., Suppl. $7: 1-473$

Macan (T.T.) 1950. - Ecology of fresh-water Mollusca in the English Lake District. J. Anim. Ecol., 19 : 124-146.

Masson (J.P.), Rouault (J.V.) \& Vidonne (A.). 1978. - Le lac de Narlay (Jura). Evolution de quelques paramètres physicochimiques en relation avec la présence d'Oscillatoria rubescens. In: Actes du colloque A.F.L. sur les lacs naturels, 18 an 21 septembre 1978 , Chambéry. Univ. Savoie, C.T.G.R.E.F. ed. : 97-114.

Meier-Brook (C.). 1963. - Ober die Mollusken der Hochschwarzwald- und Hochvogesen Gewässer. Arch. Hydrobiol., Suppl. $28(5): 1-46$

Monod (R.), Blanc (P.) \& Corvi (C.). 1984. - Evolution physicochimique. In : Le Lèman, synthèse 1957-1982. Commission internationale pour la protection des eaux du Lèman contre la pol. lution. Lausanne : 89-120.

Mouthon (J.). 1980. - Contribution à l'ècologie des mollusques des eaux courantes. Esquisse biotypologique er données écologiques. Thèse $3^{*}$ cycle. Univ. Paris VI, $169 \mathrm{p}$.

Mouthon (J.). 1981 a. - Les Mollusques et la pollution des eaux douces : ébauche d'une gamme de polluosensibilité des espèces. Bijdrogen to de Die rkande, 5 l (2) : 250-258.

Mouthon (J.). 1981 b. - Les Mollusques des lacs de l'Abbaye et des Rousses (Massif du Jura). Ann. Scient. Univ. Besançon, 4 (2): 1 -25.

Mouthon (J.) 1986. - Contribution à la connaissance des Mollusques du Lac léman. Intérêt de l'étude des malacocénoses pour apprécier la qualité biologique des sédiments de ce plan d'eau (sous presse).

Murray (J.) \& Pullar (L.). 1910. - Bathymetrical survey of the freshwater lochs of Scotland. Edinburgh : Challenger. Vols 1-6.

Ruggiu (D.) \& Saraceni (C.). 1972. - A statistical study of the distribution and abundance of the botton launa of a lake undergoing accelerate eutrophication (lake Megozzo, N. Italy). Memo rie Ist. ital. Idrobiol., $29: 169.187$

Särkkä (J). 1972. - The bottom macrofauna of the oligotrophic lake Konnevesi, Finland. Ann. Zoot. Fennici, 9: 141-146.

Slack (H.D.). 1965. - The profundal fatuna of loch Lomond, Scotland. Proc. R. Soc. Edinb., B, 69:272.297.

Steenberg (C.M.). 1917. - Furesöens Molluskfauna. In : Furesostudier C. Wesenberg-Lund. K. Danske Viudensk. Selsk. Skr. Naturv. Og. mat. Afd. 8,3 (1): 1-208.

Verneaux (J.). Vidonne (A.) \& Guyard (A.). - Caractères généraux des sediments de dix lacs jurassiens. Sciences de l'Eau (sous presse).

Vivier (P.) \& Serruya (S.). 1966. - La pollution organique du lac de Nantua. Eau, 167-171.

Wesenberg-Lund (C.). 1917. - Furesöstudie, en bathymetrisk zoologisk undersögelse of Mölleaaens șöer. D.K.D. Vidensk, Selsk, $S k$ r., naturvidensk. of methom. Ajd., $8(1): 1.208$.

Zschokke (F.). 1911. - Die Tiefseefauna der Seen Mitteleuropas. Int. Revue ges. Hydrobiol. Hydrogr., 4 : 1-246.

Rapport C.T.G.R.E.F. 1979. - Etude ecologique des lacs des Vosges Géraramer et Longemer. Etude $\mathrm{n}^{\circ} 44: 79 \mathrm{p}+$ annexes.

Rapport S.R.A.E. Franche-Comtè. 1984. - Les locs de Clairvaux. Monographies écologiques. $99 \mathrm{p}$. + annexes.

Rapport S.R.A.E. Franche-Comté. 1986. - Le lac de Chalain. Etude écologique (sous presse). 